\title{
Resource Reservation Protocol and Fast Reroute Mechanism for Implementing Traffic Engineering In Multiprotocol Label Switching Networks
}

\author{
Venkateswari $\mathrm{R}^{1}$, Ravindran $\mathrm{M}^{2}$, Kalai $\mathrm{K} \mathrm{N}^{3}$ \\ \{rvenkateswari@gmail.com ${ }^{1}$, mravibsnl@gmail.com ${ }^{2}$, kalaikn13@gmail.comaddress ${ }^{3}$ \} \\ Associate Professor, Department of Electronic and Communication Engineering. PSG college of \\ technology, Coimbatore,India ${ }^{1}$, AGM, BSNL, RGM-TTC, GST Road, Meenambakkam, Chennai, India ${ }^{2}$, \\ Department of Electronic and Communication Engineering. PSG college of technology, \\ Coimbatore, India $^{3}$
}

\begin{abstract}
Multiprotocol Label Switching commonly referred to as MPLS was the talk in the networking industry but now it is a widely deployed technology in the service provider networks. MPLS is a very effective solution to explain the various problems faced by present-day networks that include scalability, speed, management of quality of service (QoS) and also traffic engineering. It also provides an effective solution to satisfy the management of bandwidth and service requirements for the next-generation IP-based backbone networks. With the help of MPLS core network the Resource Reservation Protocol (RSVP) has been used to create Traffic Engineering (TE) Tunnels. RSVP is a transport layer protocol which reserves some bandwidth along a route from a particular source to destination. It is predominantly used for the quick delivery of the transmission packets by the receiver side from the sender to the receiver. RSVP can be referred to as a signaling protocol that reserves some resources, for the purpose of IP unicast and multicast flows, and requests for QoS parameters for several applications. The fast reroute mechanism has been implemented in this project, for redundancy purpose. With the help of this mechanism the packets are being delivered to the customer at the receiver end with high speed and performance. In this project the drawbacks faced by the MPLS core network using the Label Distribution Protocol (LDP) have been overcome by using the RSVP. It was observed that the latency is reduced in RSVP by $10 \mathrm{~ms}$ for smaller sized packets (100) and by around 20ms for larger sized packets (10000) when compared to LDP.
\end{abstract}

Keywords: MPLS, RSVP, Traffic Engineering.

\section{Introduction}

Multiprotocol Label Switching commonly referred to as MPLS was the talk in the networking area, a while ago, and now it is a widely used technology in the service provider networks. MPLS is one of the most effective solutions to explain the various problems faced by present-day networks: scalability, speed, management of quality of service (QoS) and also traffic engineering. It also provides an effective solution to satisfy the management of bandwidth and service requirements for the next-generation IP-based backbone networks. The MPLS is hence a very good solution for several problems in networking. 
From the last two years, the Internet has become a network that is being used everywhere and has also inspired the development of many new applications in the consumer and business markets. But, in terms of bandwidth and speed, the demands that are placed by these new services and applications on the network, have wanted more of the resources than the expected level of the already existing web network. This network change towards a packet-and cellbased network has introduced a lot of uncertainty into the so-called deterministic network. Apart from the issue of these resource constraints, there is another challenge relates to the transportation of both the bits as well as the bytes over the backbone in order to provide a good differentiation for classes of service to users.

The volume of traffic and the number of users that grow exponentially add to this problem. Issues regarding Quality of Service and Class of service $(\mathrm{CoS})$ need to be taken care of in order to satisfy the diverse necessities of the vast range of users of the network. MPLS plays an important role in the routing of packets through the network, switching them across the network, and also forwarding of these packets through the network in order to meet the service requirements of the network users.

With the help of MPLS core network the Resource Reservation Protocol (RSVP) has been used to create Traffic Engineering (TE) Tunnels. RSVP is a transport layer protocol which reserves some bandwidth along a route from a particular source to destination. It is predominantly used for the quick delivery of the transmission packets by the receiver side from the sender to the receiver.

The fast reroute mechanism has been implemented in this project, for redundancy purpose. With the help of this mechanism the packets are being delivered to the customer at the receiver end with high speed and performance.

In this project the drawbacks faced by the MPLS core network using the Label Distribution Protocol (LDP) have been overcome by using the RSVP.

The existing methodologies are discussed in Section II. Section III deals with the system proposed in the project. The Results and Analysis that has been done are discussed in Section IV. Section V finally concludes the entire project.

\section{Existing System}

Multi-Protocol Label Switching (MPLS) depends on label switching which is for reliable packet delivery in an ongoing internet service. Here the TE algorithm is designed based on the operator choices. LDP requires much less configuration, generates less control traffic and requires fewer labels for setting up full LSP meshes. However, since LDP does not have the ability to setup explicitly routed paths it is not possible to provide traffic engineering or traffic protection with LSPs setup by LDP. In certain cases, LDP can be used for traffic engineering but it results in excessive network complexity. A further complication with LDP is its dependence on the IGP; when the IGP re-converges after a network change the temporary inconsistencies in the IGP next hops may be propagated to LDP and the MPLS forwarding plane may suffer temporary traffic disruptions. Some other drawbacks are:

The best and shortest route was over used which resulted in heavy network traffic and also that some links were left unused which further caused wastage of bandwidth.

LDP maintains an IGP convergence timer that is set to an IGP convergence interval each time an IGP change event is received. LDP takes some time to converge again. If there is another IGP failure before LDP has converged MPLS forwarding could be disrupted. 
The packets that were lost were unable to retrieve in many cases. Auto TE is an efficient and applicable solution for traffic engineering decisions but resulted in delay.

If at some point traffic encounters another failure and since there are no backup next hops, traffic will be dropped and there will be no traffic loops. Router failures will be handled in the same way since it is assumed that when a router fails, all its adjacent links fail.

\section{Proposed System}

In order to overcome the drawbacks mentioned earlier in the previous section, a better approach is used and more focus is given on the effectiveness of traffic engineering. Here RSVP is used in the place of LDP.

If there are any link failures during the RSVP-TE operation, tunnels can be used. By using tunnels, efficient data transfer without any delay can be ensured. Various reservation styles are provided by RSVP that allow future styles to be added to protocol revisions in order to fit into many varied applications. Using RSVP also minimizes the packet loss and network traffic as each one of them have their own reserved links. Efficient routing can be achieved when RSVP is used along with the IPv6. Better quality of service (QOS) can be provided.

Even when the recovery mechanisms for network links have been implemented at the IP layer, its reinstallation takes a lot of time, up to several seconds which is not acceptable when it comes to real time applications. So here fast reroute mechanism is been implemented which helps in fast recovery.

\section{Results And Analysis}

\section{A. LDP vs. RSVP}

To analyse the differences and to find the efficient protocol, Round Trip Time (RTT), Latency and Throughput was noted for three different cases.

Round Trip Time (RTT): It is the length of time that it takes for one signal to be sent added to the length of time it takes for an acknowledgement of that signal to be received.

Latency: It refers to time interval or delay in which a system component is waiting for yet another system component in order to do something. This duration of time is referred to as latency. Formula: Latency $=\mathrm{RTT} / 2$

Throughput: It is defined as the amount of information or material passed put through or delivered in a specific period of time. Formula: Throughput = Packet Size / Latency

$$
\text { 1) Case } 1
$$

For a total of 7 different Packet Sizes and with 25 repetitions of each of the packet sizes, the readings with MPLS network using Label Distribution Protocol and Resource Reservation Protocol were observed.

Core Router: R1 To R2

In the first case, the packet transfer from Core Router R1 to R2 is considered. Table I depicts the RTT in ms, Latency in ms and the throughput for LDP from core router R1 to R2. Table II depicts the RTT in ms, Latency in $\mathrm{ms}$ and the throughput for RSVP from core router $\mathrm{R} 1$ to $\mathrm{R} 2$. 


\begin{tabular}{|l|l|l|l|}
\hline Packet size & RTT(ms) & Latency(ms) & Throughput \\
\hline 100 & 42 & 21 & 4.76 \\
\hline 500 & 46 & 23 & 21.73 \\
\hline 1000 & 52 & 26 & 38.46 \\
\hline 2000 & 72 & 36 & 55.55 \\
\hline 2500 & 91 & 45.5 & 60 \\
\hline 5000 & 139 & 69.5 & 71.94 \\
\hline 10000 & 205 & 102.5 & 97.56 \\
\hline
\end{tabular}

TABLE II. RSVP- Core Router R1 to R2

\begin{tabular}{|l|l|l|l|}
\hline Packet size & RTT(ms) & Latency(ms) & Throughput \\
\hline 100 & 30 & 15 & 6.66 \\
\hline 500 & 37 & 18.5 & 27.02 \\
\hline 1000 & 44 & 22 & 45.45 \\
\hline 2000 & 55 & 27.5 & 72.72 \\
\hline 2500 & 91 & 45.5 & 54.94 \\
\hline 5000 & 110 & 55 & 90.90 \\
\hline 10000 & 169 & 84.5 & 118.34 \\
\hline
\end{tabular}

The RTT in ms with respect to the packet size for both LDP and RSVP from core router R1 to R2 are plotted in the graph shown in Figure 1. It can be observed from the graph that as the number of packets increase, the RTT also increases in both LDP and RSVP. But it can be observed that the RTT for RSVP is less compared to LDP while considering two similar cases. The reason for this reduction in the total round trip time in RSVP is the implementation of traffic engineering using the fast reroute mechanism. It is observed that the latency is reduced in RSVP by $10 \mathrm{~ms}$ for smaller packets (100) and by around $20 \mathrm{~ms}$ for larger packets (10000) when compared to LDP. This can be clearly observed from the graph.

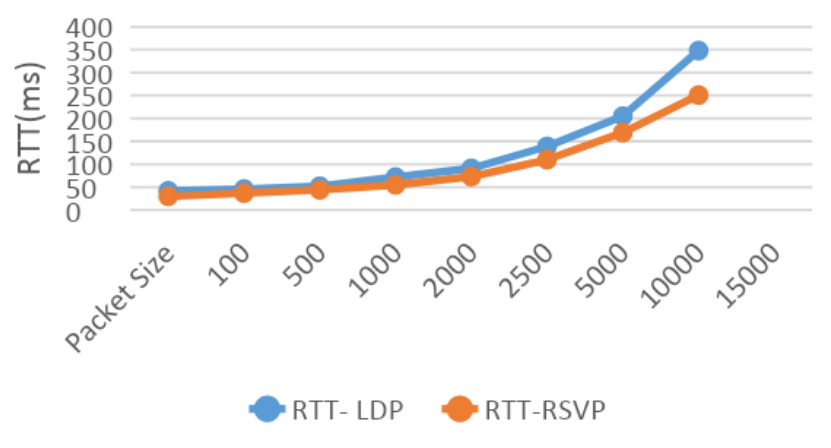

Fig. 1. Graph for R1-R2 Values

2) Case 2

PE-PE Customer A 
In the second case, the packet transfer from provider edge router to provider edge router in Customer A is considered. Table III depicts the RTT in ms, Latency in $\mathrm{ms}$ and the throughput for LDP from provider edge router to provider edge router in Customer A. Table IV depicts the RTT in ms, Latency in $\mathrm{ms}$ and the throughput for RSVP from provider edge router to provider edge router in Customer A.

TABLE III.

LDP- PE-PE Customer A

\begin{tabular}{|l|l|l|l|}
\hline Packet Size & RTT(ms) & Latency(ms) & Throughput \\
\hline 100 & 91 & 45.5 & 2.19 \\
\hline 500 & 114 & 57 & 8.77 \\
\hline 1000 & 129 & 64.5 & 15.50 \\
\hline 2000 & 140 & 70 & 28.57 \\
\hline 2500 & 147 & 73.5 & 34.01 \\
\hline 5000 & 211 & 105.5 & 47.39 \\
\hline 10000 & 308 & 154 & 64.93 \\
\hline
\end{tabular}

The RTT in ms with respect to the packet size for both LDP and RSVP from provider edge router in Customer A are plotted in the graph shown in Figure 2. It can be observed from the graph that as the number of packets increase, the RTT also increases in both LDP and RSVP. But it can be observed that the RTT for RSVP is less compared to LDP while considering two similar cases. The reason for this reduction in the total round trip time in RSVP is the implementation of traffic engineering using the fast reroute mechanism. It is observed that the latency is reduced in RSVP by $10 \mathrm{~ms}$ for smaller packets (100) and by around 20ms for larger packets (10000) when compared to LDP. This can be clearly observed from the graph.

\begin{tabular}{|l|l|l|l|}
\multicolumn{2}{c}{ TABLE IV. } & RSVP- PE-PE Customer A \\
\hline Packet Size & RTT(ms) & Latency(ms) & Throughput \\
\hline 100 & 74 & 37 & 2.70 \\
\hline 500 & 93 & 55.5 & 9.00 \\
\hline 1000 & 111 & 45.5 & 21.97 \\
\hline 2000 & 133 & 87.5 & 22.85 \\
\hline 2500 & 139 & 86 & 29.06 \\
\hline 5000 & 189 & 114.5 & 43.66 \\
\hline 10000 & 268 & 154.5 & 64.72 \\
\hline
\end{tabular}




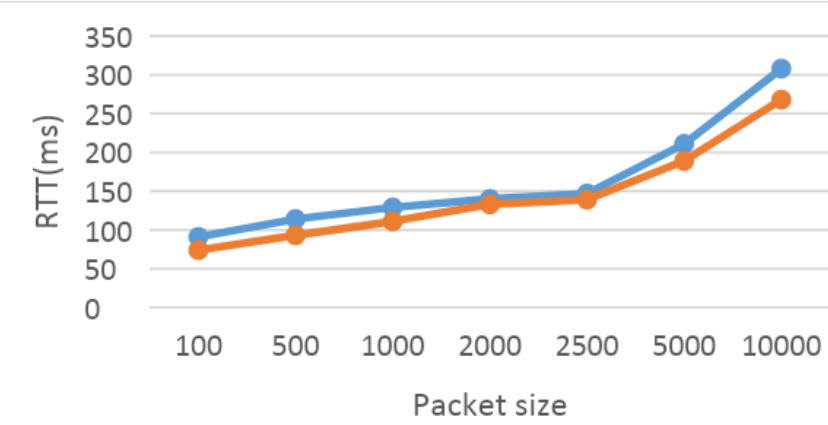

RTT-LDP $\quad$ RTT-RSVP

Fig. 2. Fig 4.2 Graph for PE-PE Values Customer A 3) Case 3:

PE-PE Customer B

In the third case, the packet transfer from provider edge router to provider edge router in Customer B is considered. Table V depicts the RTT in ms, Latency in ms and the throughput for LDP from provider edge router to provider edge router in Customer B. Table VI depicts the RTT in ms, Latency in ms and the throughput for RSVP from provider edge router to provider edge router in Customer B.

TABLE V. LDP- PE-PE Customer B

\begin{tabular}{|l|l|l|l|}
\hline Packet Size & RTT(ms) & Latency(ms) & Throughput \\
\hline 100 & 96 & 48 & 2.08 \\
\hline 500 & 108 & 54 & 9.25 \\
\hline 1000 & 124 & 62 & 16.12 \\
\hline 2000 & 133 & 66.5 & 30.07 \\
\hline 2500 & 137 & 68.5 & 36.49 \\
\hline 5000 & 235 & 117.5 & 42.55 \\
\hline 10000 & 331 & 165.5 & 60.42 \\
\hline \multicolumn{4}{|c|}{ RSVP- PE-PE Customer B } \\
\hline Packet Size & TABLE VI. & Latency(ms) & Throughput \\
\hline 100 & 67 & 33.5 & 2.98 \\
\hline 500 & 91 & 45.5 & 10.98 \\
\hline 1000 & 101 & 50.5 & 19.80 \\
\hline 2000 & 121 & 60.5 & 33.05 \\
\hline 2500 & 130 & 65 & 38.46 \\
\hline 5000 & 222 & 111 & 45.04 \\
\hline 10000 & 291 & 145.5 & 68.72 \\
\hline
\end{tabular}

The RTT in ms with respect to the packet size for both LDP and RSVP from provider edge router in Customer B are plotted in the graph shown in Figure 3. It can be observed from the graph that as the number of packets increase, the RTT also increases in both LDP and RSVP. But it can be observed that the RTT for RSVP is less compared to LDP while considering two similar cases. The reason for this reduction in the total round trip time in RSVP is the implementation of traffic engineering using the fast reroute mechanism. It is 
observed that the latency is reduced in RSVP by $10 \mathrm{~ms}$ for smaller packets (100) and by around 20ms for larger packets (10000) when compared to LDP. This can be clearly observed from the graph.

From the results and analysis obtained above, some conclusions and inferences were drawn about the advantages of RSVP over LDP that will be discussed in the next sub section.

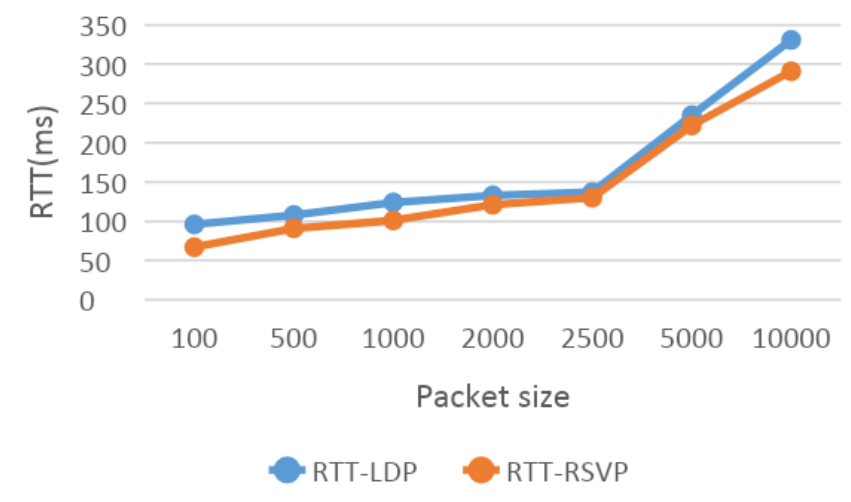

Fig. 3. Graph for PE-PE Customer B

B. Inference

TABLE VII. LDP vs RSVP

\begin{tabular}{|l|l|}
\hline Label Distribution Protocol & Resource Reservation Protocol \\
\hline $\begin{array}{l}\text { In the case of Label Distribution Protocol, the label- } \\
\text { switched path is initiated by the egress. }\end{array}$ & $\begin{array}{l}\text { In Resource Reservation Protocol, } \\
\text { the label-switched path is initiated by } \\
\text { the ingress. }\end{array}$ \\
\hline The LDP is the automatic pilot. & $\begin{array}{l}\text { On the other hand, RSVP requires } \\
\text { configuration }\end{array}$ \\
\hline It is easy to configure. & It is of complex configuration. \\
\hline Data transfer is slower when compared to RSVP. & Data transfer is faster in RSVP. \\
\hline $\begin{array}{l}\text { With Label Distribution Protocol, the label-switched } \\
\text { paths from different ingresses share the same label in } \\
\text { order to go to the egress, in other words, called the } \\
\text { multi-point to point label switched paths. }\end{array}$ & $\begin{array}{l}\text { On the other hand, Resource } \\
\text { Reservation Protocol sets up the } \\
\text { independent point to point label } \\
\text { switched paths. }\end{array}$ \\
\hline $\begin{array}{l}\text { The Label Distribution Protocol is not suitable for } \\
\text { traffic engineering and resource reservations. }\end{array}$ & $\begin{array}{l}\text { RSVP can efficiently handle traffic } \\
\text { engineering and resource } \\
\text { reservations. }\end{array}$ \\
\hline QoS mechanism is limited. & $\begin{array}{l}\text { QoS mechanism is a guaranteed } \\
\text { resource in RSVP. } \\
\text { Bandwidth Reservation is required. }\end{array}$ \\
\hline Bandwidth reservation is not required. & \multicolumn{1}{|c|}{ and } \\
\hline
\end{tabular}

From the discussions in the previous sections, some inferences about the advantages of RSVP over LDP were analyzed. It was observed that the latency is reduced in RSVP by 10ms for smaller packets (100) and by around 20ms for larger packets (10000) when compared to LDP.Table VII shows the different advantages of RSVP over LDP that were analyzed. In 
RSVP, the RTT is less when compared to LDP as RSVP as Fast reroute mechanism is implemented in RSVP.

From the comparison between LDP and RSVP shown in Table VII, it can be concluded that if there is a need to set up label-switched paths, then Label Distribution Protocol can used. If traffic engineering and resources reservations are necessary, then Resource Reservation Protocol along with Traffic Engineering has to be used for its distinctive characteristics that support the mechanism of Traffic Engineering.

\section{Applications and Future Scope}

It provides improved packet-forwarding performance within the network. MPLS also simplifies and enhances packet forwarding through routers using the Layer-2 switching paradigms. It also increases the performance of the network as it helps in enabling routing by switching at wireline speeds. It also supports quality of service differentiation. It also uses a traffic-engineered path setup that helps to achieve service-level guarantees. It also incorporates various provisions for a constraint-based and an explicit path setup. It helps in network scalability. MPLS helps in building scalable VPNs along with traffic-engineering capability.

The usage of Multi-Protocol Label Switching to manage bandwidth in the future mobile wireless network is both profitable and reliable because of its valuable cost to the both operators and also service providers. This results in providing sustainable quality of services to our users. One other critical problem is delays such as queue delays, end-to-end delay and packet delay. With the Multi-Protocol Label Switching, these delays would be reduced to a great extent. But it would become an addition to the expenses in order to implement the MultiProtocol Label Switching technology to a network that is already in existence, instead of getting rid of the existing IP technology together along with the complete facilities. The future scope of this paper is that it could be expanded by creating an algorithm that is capable of measuring both Class of service and quality of service. Performance of the quality of service schemes such Priority Queuing, First in First out and Weighted Fair Queuing should undergo evaluation and then be employed in order to assess the services that can be provided to the consumers. Furthermore analysis on the Multi-protocol Label Switching- traffic engineering could be considered for giving sufficient amount of bandwidth to the mobile as well as the wireless networks of the future. The model that has been designed will require more amount of verification, validation and also more refinement in order to meet the requirements of minimum bandwidth and the data rates specifications for the $5 \mathrm{G}$ technology.

\section{Conclusion}

Multi-Protocol Label Switching reduces the complexities in the network infrastructure by making data, video and voice better. Multi-Protocol Label Switching gives a much greater amount of security and also a very high availability. Through the above-mentioned analysis it can be seen that the MPLS is much faster than the traditional routing technique. If hardware facilities and software platform can be improved by real-time routers then a significant difference can be noticed. Also, in a certain event of failure of a link in the network, when recovery mechanisms are, at that point of time, being used in Internet Protocol layer, then, reinstallation takes up to many seconds that is a very large amount of time real-time application and cannot be accepted. Hence, the concept of fast rerouting in the Multi-Protocol Label Switching practically satisfies the needs of real-time application with fast recovery. 
Continuing advances in technology will result in changes in the way traffic engineering is performed in the Internet. For example, the emergence of an intelligent optical internet networking system in the future, with sophisticated bandwidth provisioning capabilities and dynamic wavelength routing based on MPLS will have a great impact on traffic engineering in the core IP networks. Coupled with these are fundamental research and development issues that remain unexplored in constraint-based routing, policy-based management of MPLS networks, CNM, and IP over optical architectures and interconnection models utilizing MPLS. The RTT becomes lesser as MPLS and Traffic Engineering are implemented. It is observed that the latency is reduced in RSVP by $10 \mathrm{~ms}$ for smaller packets (100) and by around $20 \mathrm{~ms}$ for larger packets (10000) when compared to LDP.When Traffic Engineering is implemented, a significant fall is observed at value 3 before rising again for the rest of the values. A mathematical analysis could be made as to why such a steep fall and rise is observed

\section{References}

[1] Vassilis Foteinos, Kostas Tsagkaris, Pierre Peloso, Laurent Ciavaglia, and Panagiotis Demestichas, "Operator-Friendly Traffic Engineering in IP/MPLS Core Networks", IEEE

[2] N. Wang, K. Ho, G. Pavlou, and M. Howarth, "An overview of routing optimization for internet traffic engineering,” IEEE Commun. Surveys Tuts., vol. 10, no. 1, pp. 36-56, 2008.

[3] Bringing Traffic Engineering and Resiliency to LDP Provisioned MPLS Forwarding Planes, George Apostolopoulos,ICS-FORTH, Greece.

[4] A Study of Performance Analysis of Signaling Protocols in MPLS. 3rd IEEE International Conference on "Computational Intelligence and Communication Technology" (IEEE-CICT 2017)

[5] K. Tsagkaris et al., "Operator-driven Framework for Establishing and unifying autonomic network and service management solutions," in Proc. IEEE GLOBECOM Workshops, 2011, pp. 684-689.

[6] M. Pickavet et al., "Worldwide energy needs for 1CT: The rise of power-aware networking," in Proc. ANTS Conf., Bombay, India, Dec. 15-17,2008, pp. 1-3.

[7] Cisco Press Release Annual Cisco Visual Networking Index Forecast Projects Global IP Traffic to Increase More Than Fourfold by 2014. 